OPEN ACCESS

Edited by: Andrea Ladányi, National Institute of Oncology (NIO), Hungary

*Correspondence: Cuijian Zhang surgeon_zhang@126.com

Received: 19 July 2021 Accepted: 07 September 2021 Published: 27 September 2021

Citation:

Zhang C, Qian J, Wu Y, Zhu Z, Yu W,

Gong Y, Li X, He Z and Zhou L (2021) Identification of Novel Diagnosis Biomarkers for Therapy-Related Neuroendocrine Prostate Cancer. Pathol. Oncol. Res. 27:1609968. doi: 10.3389/pore.2021.1609968

\section{Identification of Novel Diagnosis Biomarkers for Therapy-Related Neuroendocrine Prostate Cancer} Cuijian Zhang*, Jinqin Qian, Yucai Wu, Zhenpeng Zhu, Wei Yu, Yanqing Gong, Xuesong Li,
Zhisong He and Liqun Zhou

Department of Urology, Peking University First Hospital Institute of Urology, National Urological Cancer Center, Peking University, Beijing, China

Background: Therapy-related neuroendocrine prostate cancer (NEPC) is a lethal castration-resistant prostate cancer (CRPC) subtype that, at present, lacks wellcharacterized molecular biomarkers. The clinical diagnosis of this disease is dependent on biopsy and histological assessment: methods that are experience-based and easily misdiagnosed due to tumor heterogeneity. The development of robust diagnostic tools for NEPC may assist clinicians in making medical decisions on the choice of continuing antiandrogen receptor therapy or switching to platinum-based chemotherapy.

Methods: Gene expression profiles and clinical characteristics data of 208 samples of metastatic CRPC, including castration-resistant prostate adenocarcinoma (CRPC-adeno) and castration-resistant neuroendocrine prostate adenocarcinoma (CRPC-NE), were obtained from the prad_su2c_2019 dataset. Weighted Gene Co-expression Network Analysis (WGCNA) was subsequently used to construct a free-scale gene co-expression network to study the interrelationship between the potential modules and clinical features of metastatic prostate adenocarcinoma and to identify hub genes in the modules. Furthermore, the least absolute shrinkage and selection operator (LASSO) regression analysis was used to build a model to predict the clinical characteristics of CRPC-NE. The findings were then verified in the nepc_wcm_2016 dataset.

Results: A total of 51 co-expression modules were successfully constructed using WGCNA, of which three co-expression modules were found to be significantly associated with the neuroendocrine features and the NEPC score. In total, four novel genes, including NPTX1, PCSK1, ASXL3, and TRIM9, were all significantly upregulated in NEPC compared with the adenocarcinoma samples, and these genes were all associated with the neuroactive ligand receptor interaction pathway. Next, the expression levels of these four genes were used to construct an NEPC diagnosis model, which was successfully able to distinguish CRPC-NE from CRPC-adeno samples in both the training and the validation cohorts. Moreover, the values of the area under the receiver operating characteristic (AUC) were 0.995 and 0.833 for the training and validation cohorts, respectively. 
Conclusion: The present study identified four specific novel biomarkers for therapyrelated NEPC, and these biomarkers may serve as an effective tool for the diagnosis of NEPC, thereby meriting further study.

Keywords: biomarker, WGCNA, signature, neuroendocrine prostate cancer (NEPC), LASSO, mCRPC

\section{INTRODUCTION}

Prostate cancer $(\mathrm{PCa})$ is one of the most prevalent cancers for men in western countries and throughout the world with an estimated incidence of 1.276 million cases in 2018. Owing to the aging population globally, the incidence rate of $\mathrm{PCa}$ is expected to increase to 2.3 million by the year 2040, thereby posing an even greater threat to human health $[1,2]$. Although the 5 years survival rate of localized $\mathrm{PCa}$ is $>95 \%$, the overall survival time for the advanced stage of this disease, metastatic castration-resistant prostate cancer (mCRPC), is only 9-36 months [3]. Moreover, nearly $15-20 \%$ of mCRPC patients apparently undergo a histological change from the adenocarcinoma to the neuroendocrine subtype following multiple treatments, which is a terminally aggressive PCa subtype [4]. The emergence of neuroendocrine prostate cancer (NEPC) may result in resistance to the androgen receptor (AR)based therapies, a development that is significantly unfavorable towards patients' survival [5]. Meanwhile, NEPC patients may develop additional sensitivity to platinum-based chemotherapy, which is the recommended therapeutic intervention to treat NEPC according to the National Comprehensive Cancer Network (NCCN) guidelines [6]. The timely diagnosis of NEPC prevents patients from being subjected to ineffective standard treatments of CRPC, including novel hormone and Taxane-based therapies, and also enables the progression of the disease to be effectively indicated, even in the absence of (or with an underestimated elevation of) prostate-specific antigen (PSA).

At present, the diagnosis of treatment-emergent NEPC is dependent on the histological assessment of tissue biopsies according to the World Health Organization (WHO) classification system of 2004, and neuroendocrine markers, including chromogranin A, synaptophysin (SYP), neuronspecific enolase (NSE), and CD56, are widely used. However, these diagnoses are not only experience-based but also may lead to misdiagnosis due to the presence of mixed tumors (with both adenocarcinoma and NEPC morphologies) or intratumor heterogeneity [7]. Currently, there is no robust molecular diagnosis tool available for NEPC due to its relative rarity and the limited availability of tissue samples. It is worth mentioning that a recognized diagnostic tool that addresses the NEPC scores was used to calculate the scores of a set of 70 genes, and the correlations among their alterations in DNA, RNA, and/or epigenomic status with the NEPC feature [8, 9]. However, though this tool can accurately distinguish NEPC from CRPC, its comprehensive but complex profiling methods are likely to hinder its applicability to a wider range of clinical practices. The features of NEPC include indifference towards the AR signaling pathway, downregulation of known androgen-regulated genes, and overexpression of neuroendocrine-associated genes [10, 11]. For example, co-upregulation of the LIN28B, SOX2, EZH2, and SPINK1 genes was reported in NEPC tumors [12-14]. Based on the biological findings, further studies, however, are required to combine molecular features for predicting NEPC transformation and to identify patients at a higher risk of developing lineage plasticity. Regrettably, certain of the markers that are in the process of being developed, including neuronal markers such as SYP, enolase 2 (ENO2), chromogranin-A (CHGA), and CD56 [15]; DNA methylation [16, 17]; the alterations in the expression of mRNA [12-14]; and non-coding RNAs [7] exhibit either low accuracy or are at too preliminary a stage for clinical application at the present time. Therefore, the identification of novel and robust biomarkers for the diagnosis of NEPC is urgently required.

In order to establish an effective diagnostic tool for NEPC, in the present study, Weighted Gene Co-expression Network Analysis (WGCNA) and the least absolute shrinkage and selection operator (LASSO) Cox regression analysis were performed to identify correlated gene modules and hub genes in selected modules to construct an NEPC feature-related model, which was subsequently validated in another independent cohort. A flow chart indicating a schematic representation for the approach in this study was shown in Figure 1.

\section{METHODS}

\section{Data Collection}

The mRNA expression data and corresponding clinical feature of castration-resistant prostate cancer (CRPC), including castration-resistant prostate adenocarcinoma (CRPC-adeno) and castration-resistant prostate adenocarcinoma with neuroendocrine features (CRPC-NE), were all downloaded from the cBioPortal database (https://www.cbioportal.org/). Specifically, a total of 208 CRPC samples with expression data were gained from SU2C/PCF Dream Team [9] to set as the training dataset, and 49 samples were obtained from the Neuroendocrine Prostate Cancer program (nepc_wcm 2016) [8] and applied as the validation dataset. The clinical features of the training and validation dataset were demonstrated in Table 1.

\section{Co-Expression Network Construction using WGCNA and Target Prediction}

The WGCNA algorithm was run in the $\mathrm{R}$ software package (http://www.r-project.org/) to assess the clinical characteristics of NEPC and their associated modules by calculating the correlation coefficient simultaneously with the minimum gene number of 30 in each module. A power value of 3 was used in this 


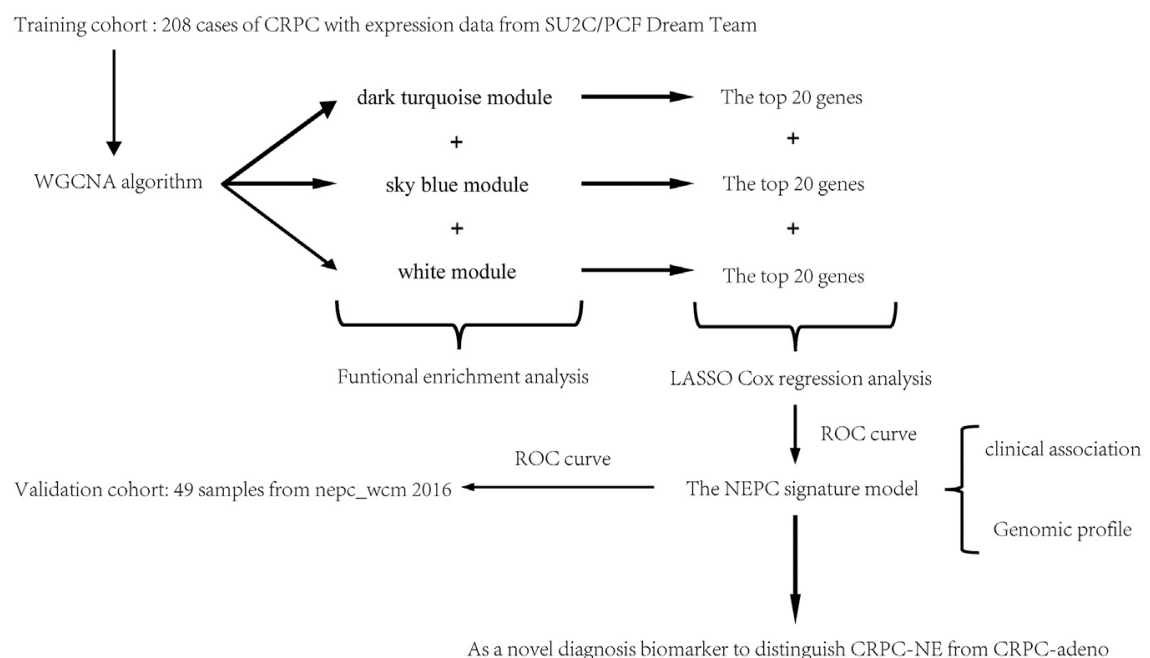

FIGURE 1 | A workflow chart for constructing an NEPC signature model.

analysis as the package suggested, and values above a predetermined threshold are considered similar. Each module can be associated with a characteristic according to the eigenvector of the module and the correlation coefficient of the phenotype or the saliency $p$-value of the module. With the application of a network heat map, the connections between the characteristic association module and other modules can be visualized. A hierarchical clustering tree was constructed based on the weighted correlation coefficients of genes and genes with similar patterns in one module.

\section{Functional Enrichment Analysis of Co-Expression Modules}

We uploaded the data to the database for Annotation, Visualization, and Integrated Discovery (DAVID) (https:// david.ncifcrf.gov/) for analyzing the function of genes in key co-expression modules which is a classic gene enrichment analysis website, mainly used for differential gene function and pathway enrichment analysis.

\section{Identification of Hub Genes in Selected Modules}

After screening the key gene modules associated with the characteristics of NEPC, the gene co-expression network map was drawn based on the relations of the genes within the modules. For each module, we selected the top 20 genes to identify the hub genes.

\section{Model Building and Validation}

LASSO, which is suitable for the regression of high-dimensional data, was used to select the most useful predictive features from the primary data set [18]. Tuning parameter (L) selection in the LASSO model used 10-fold cross-validation via minimum criteria. The NEPC signature score was calculated by the following formula: NEPC signature score $=$ gene 1 expression $\times \gamma_{1}+$ gene 2 expression $\times \gamma_{2}$ + gene 3 expression $\times \gamma_{3}+$. + gene $n$ expression $\times \gamma_{\mathrm{n}}$, where $\gamma_{\mathrm{n}}$ denotes the coefficient for each hub gene in the multivariate Cox regression model. The area under the receiver operating characteristic curve was plotted using the "timeROC" package in R studio. A coefficient profile plot was produced against the $\log (\mathrm{L})$ sequence. A

TABLE 1 | The clinical information of CRPC samples.

Variable

Total

Age

Mutation count

AR score

Genomic burden

Gleason score
SU2C/PCF Dream team

Median (range)

Median (range)

Median (range)

Median (range)

6

7

8
208

208
$60.8[38.6,80.6]$
$65[1,1,065]$
$0.481[-0.265,0.694]$
$N A$
13
46
23
65
13
1

nepc_wcm 2016

NA

NA

NA

NA

NA

NA

CRPC, castration-resistant prostate cancer, NA, not applicable. 

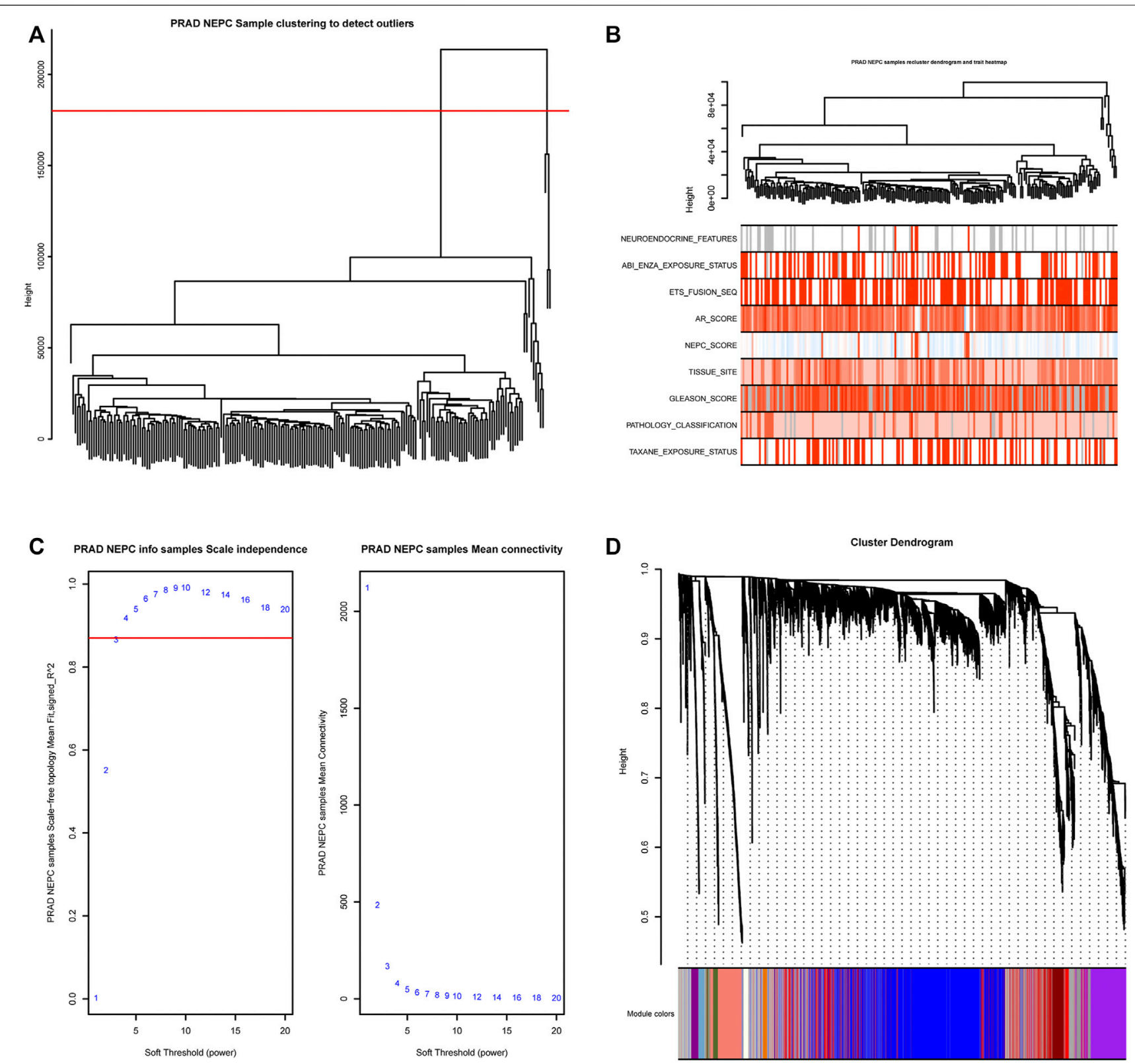

FIGURE 2 I WGCNA module clustering and soft threshold power identification. (A) Hierarchical clustering dendrogram of 208 samples based on the Euclidean distance; all samples were included in the analysis. (B) The clustering dendrogram and heat map shows the Euclidean distance and sample characteristic correlations. Light color represents a lower value, dark color represents a higher value, and grey represents a missing value. (C) Soft threshold power screening based on the network topology, the analysis used a power of 3 as the package suggested, the left panel is the coordinate map of the soft-thresholding power ( $x$-axis) and the scale-free fit index ( $y$-axis), and the right panel is the coordinate map of the mean connectivity ( $y$-axis) and the soft-thresholding power ( $x$-axis). (D) Co-expression Module clustering and identification dendrogram, 51 modules were identified and each row includes the highly correlated genes of one module.

score was calculated for each patient via a linear combination of selected genes that were weighted by their respective coefficients.

\section{Gene Set Enrichment Analysis}

The samples from the discovery dataset were divided into highand low-expression groups according to the genes' mRNA level, and the median expression level served as a cut-off value. GSEA was performed to identify the molecular feature of the founding biomarker genes [19].

\section{Statistical Analysis}

Statistical analysis was conducted with R software (v. 3.4.3, http://www.Rproject.org). Categorical variables were analyzed by use of the Fisher's exact test. Continuous variables were analyzed using Student's $t$-test for paired samples. The median value was used in this work as a cutoff to classify the patients the training and validation cohorts into high- and low-level groups. A $p$-value below 0.05 was considered statistically significant. 


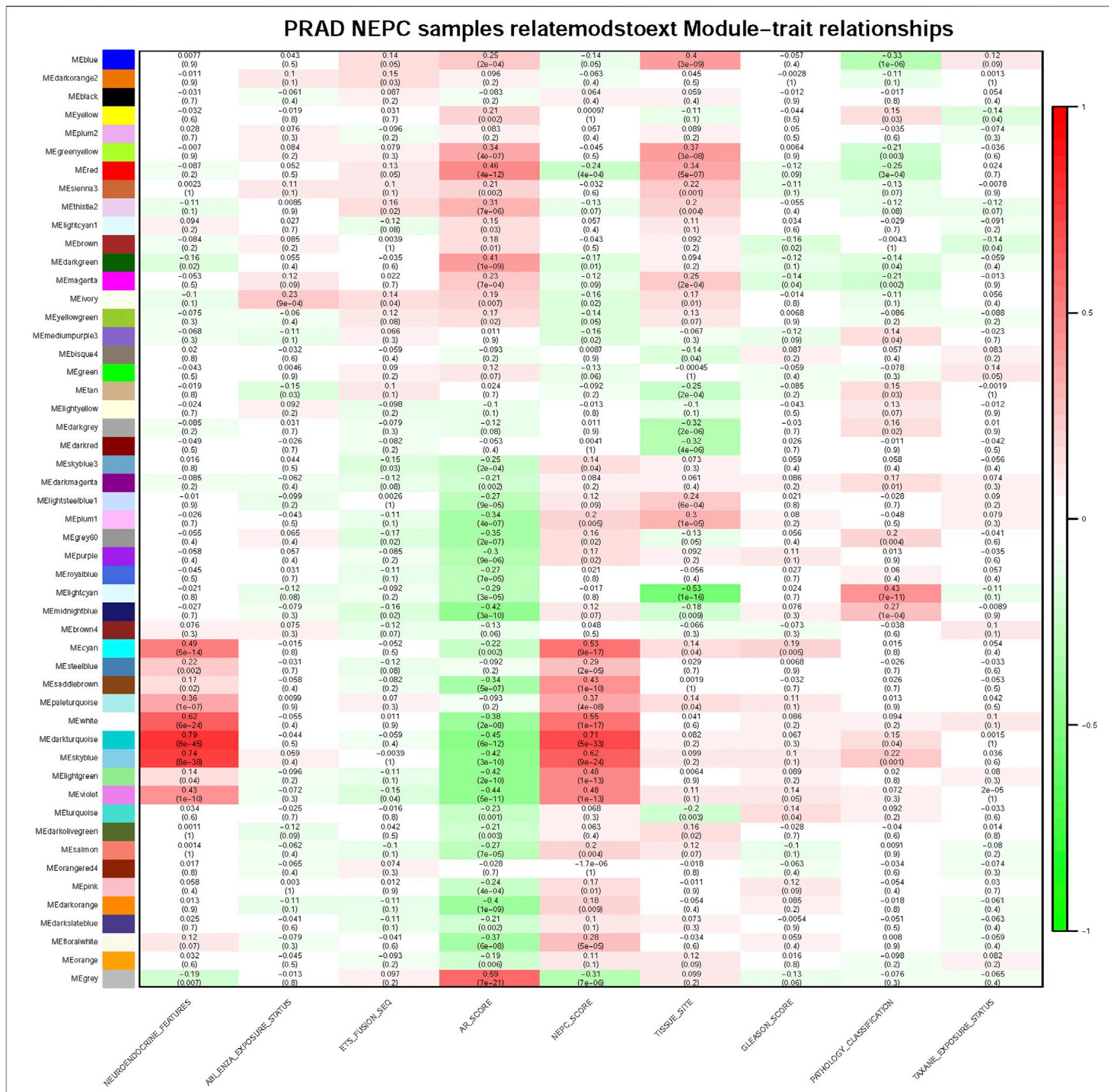

FIGURE 3 | Module and clinical characteristic relationship based on WGCNA. Module and clinical characteristic relationship heat map. In each cell, the correlation between the corresponding module and characteristic are displayed with correlation numbers and colors (red as $0.0 \sim 1.0$, green as $-1.0 \sim 0.0$ ) and $p$-values are also shown in each cell, darker color means higher correlation.

\section{RESULTS}

\section{Weighted Co-Expression Network Construction}

The gene expression matrix and clinical information of metastatic prostate adenocarcinoma samples were obtained from the SU2C-Prostate Cancer Foundation (PCF) Dream Team (Precision Therapy for Advanced Prostate Cancer). A hierarchical clustering dendrogram of 208 samples was constructed based on the Euclidean distance, all samples were included in the analysis (Figure 2A). Additionally, basic patient information, including the neuroendocrine features, the NEPC score, AR score, Gleason score, tissue site, and exposure status, amongst others, were attached below the resulting tree (Figure 2B). As recommended by the package, the soft threshold power value 3 was used to construct the gene co-expression network (Figure 2C). In total, 51 modules were identified based on co-expression 

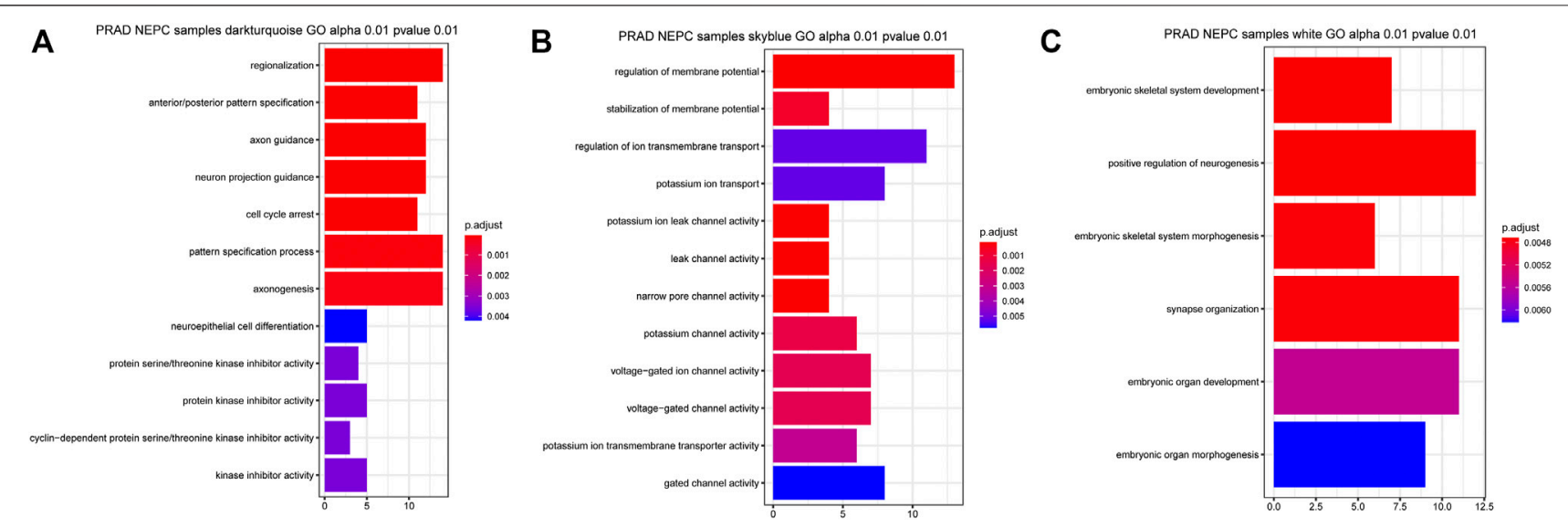

FIGURE 4 | Related signaling pathway of the three most related modules with NEPC score. (A) GO analysis of the dark turquoise module (A), sky blue module (B), and white module (C). The color indicates the significant degree of enrichment and the size indicates the number of genes enriched for each result.

module clustering and the construction of the hierarchical clustering dendrogram (Figure 2D).

\section{ASSOCIATION OF MODULES WITH CLINICAL TRAITS}

For each module, gene co-expression was summarized according to the eigengene. The correlations of each eigengene were calculated with clinical characteristics, such as Abiraterone and Enzalutamide (ABI ENZA) exposure status, AR score, tissue site, Gleason score, pathology classification, and in particular, the neuroendocrine features and NEPC score. The three highest correlated modules with the neuroendocrine feature were chosen, as denoted by the dark turquoise row $(\mathrm{r}=0.71, p<$ $0.05)$, sky blue row $(\mathrm{r}=0.62, p<0.05)$, and white row $(\mathrm{r}=0.55$, $p<0.05$ ) (Figure 3). Interestingly, each one of these three selected modules was also highly correlated with the NEPC score.

\section{ENRICHMENT ANALYSIS OF THE BIOLOGICAL FEATURES}

Gene Ontology (GO) analysis of genes in the three selected identified modules was performed, clarifying the combined features that were associated with biological processes (BP), molecular functions (MF), and cellular components (CC). The genes in the dark turquoise module were mainly associated with "regionalization", "anterior/posterior pattern specification", "axon guidance", "neuron projection guidance", "cell cycle arrest", and "pattern specification process and axon genesis" (Figure 4A). By contrast, the genes in the sky-blue module were mainly enriched in the regulation of "membrane potential", "stabilization of membrane potential", "potassium ion leak channel activity", "leak channel activity", "narrow pore channel activity", "potassium channel activity", "voltagegated ion channel activity", and "voltage-gated channel activity" (Figure 4B). Finally, the genes in the white module were involved in "embryonic skeletal system development", "positive regulation of neurogenesis", "embryonic skeletal system morphogenesis", and "synapse organization" (Figure 4C).

\section{IDENTIFICATION AND VALIDATION OF HUB GENES}

Subsequently, 314 intra-module connectivity genes were screened as candidate genes from the dark turquoise, sky-blue, and white modules. The top 60 genes were selected (Supplementary Table S1) for LASSO regression analysis, comprising the top 20 hub genes from each of the three selected modules. In total, four potential hub genes, including neuronal pentraxin 1 (NPTX1), proprotein convertase subtilisin/kexin type 1 (PCSK1), ASXL transcriptional regulator 3 (ASXL3), and tripartite motif-containing 9 (TRIM9) were identified as non-zero coefficients in the LASSO logistic regression model used to predict NEPC feature (Figures 5A, B). As shown in Figures $\mathbf{5 C}-\mathbf{F}$, these four identified genes were all associated with the neuroactive ligand-receptor interaction and olfactory transduction pathways. With the exception of PCSK1, the other three genes (NPTX1, ASXL3, and TRIM9) were all associated with complement and coagulation cascades. Additionally, PCSK1 was also associated with the calcium signaling pathway.

Through the analysis of the NEPC features in conjunction with the expression levels of the hub genes, it was demonstrated that NPTX1, PCSK1, ASXL3, and TRIM9 were all significantly upregulated in samples with NEPC feature $(p<0.001$, Figures 6A-D). With the exception of PCSK1, the expressions of the other three genes (NPTX1, ASXL3, and TRIM9) were all positively correlated with the traditional NEPC score (Figures 6E-H). Based on the aforementioned results, the NEPC signature was established by the expression of these four hub genes, consisting of NPTX1, PCSK1, ASXL3, and TRIM9 (Table 2).

In addition, the predicted signature model was applied in the training dataset, and this analysis demonstrated that the CRPCNE samples had a significantly higher predicted signature score, compared with the CRPC-adeno samples (Figure 7A). The AUC 
A

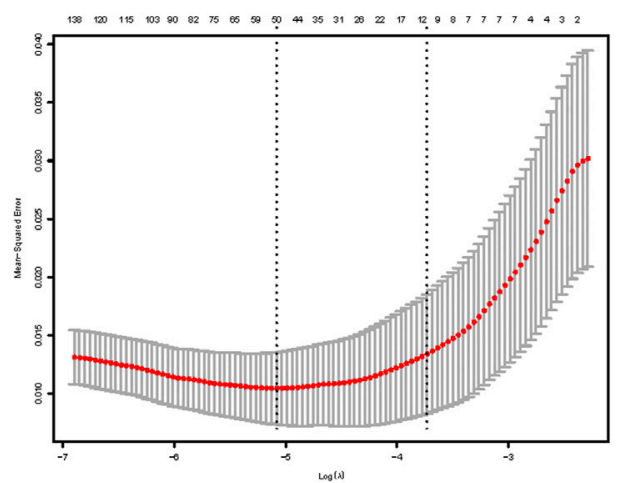

C

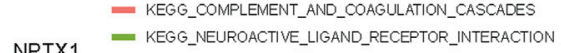
NPTX1 KEGG_OLFACTORY_TRANSDUCTION

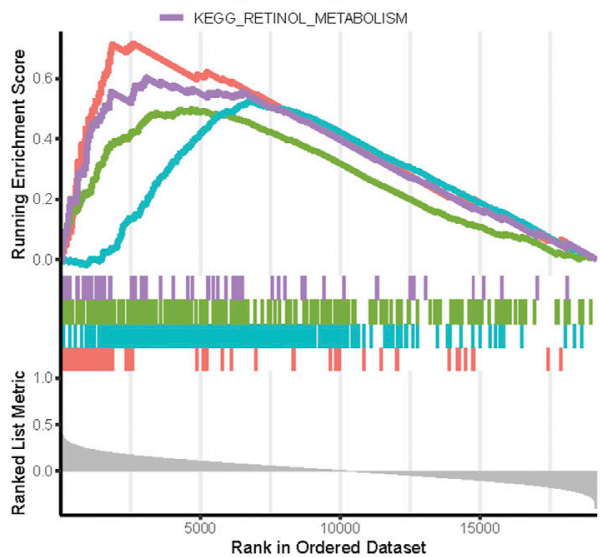

E
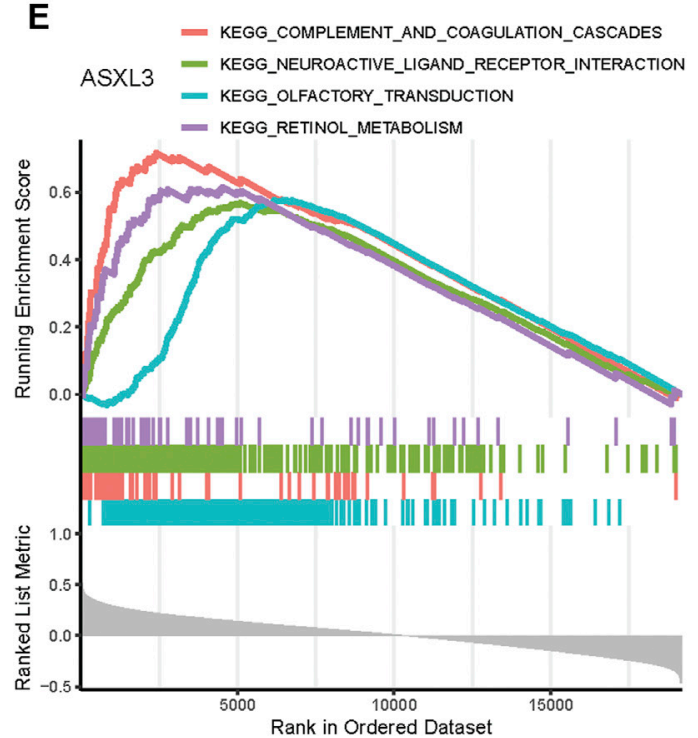

B

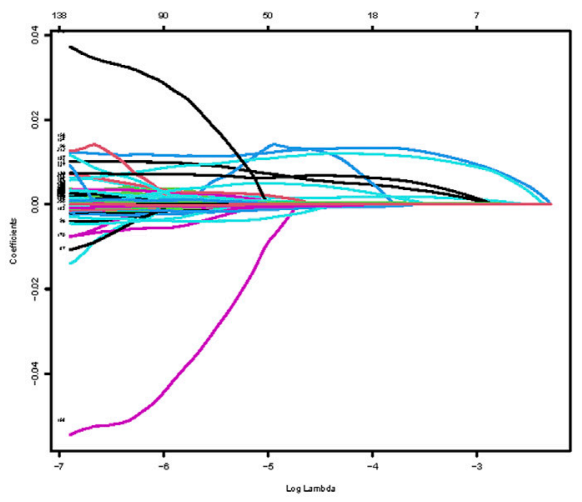

D $\quad$ KEGG_CALCIUM_SIIGNALING_PATHWAV

PCSK1 - KEGG_NEUROACTIVE_LIGAND_RECEPTOR_INTERACTION - KEGG_OLFACTORY_TRANSDUCTION

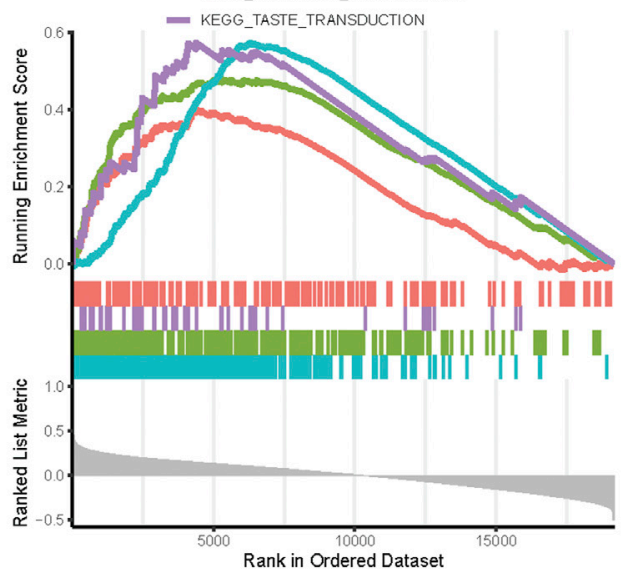

F $\quad$ KEGG_COMPLEMENT_AND_COAGULATION_CASCADES TRIM9 $=$ KEGG_CYTOKINE_CYTOKINE_RECEPTOR_INTERACTION - KEGG_NEUROACTIVE_LIGAND_RECEPTOR_INTERACTION - KEGG_OLFACTORY_TRANSDUCTION

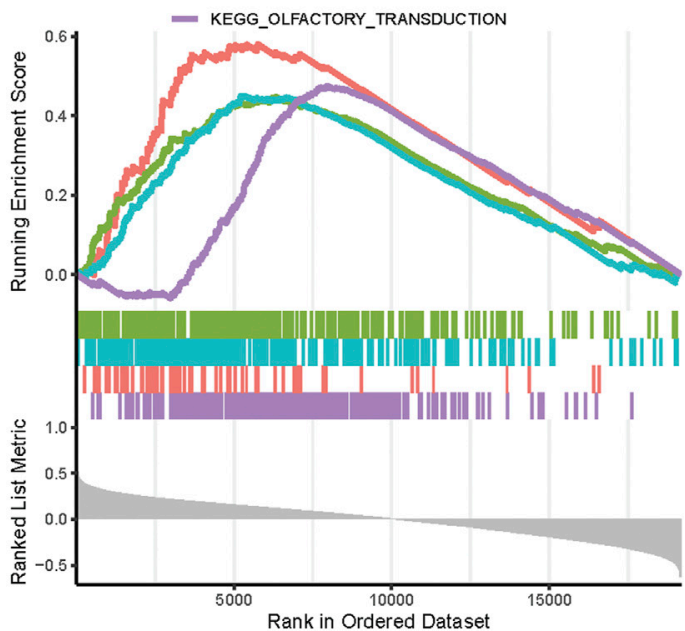

FIGURE 5 | Feature genes selection using the least absolute shrinkage and selection operator (LASSO) binary logistic regression model. (A) The result of LASSO regression analysis. (B) LASSO coefficient profiles of the 60 genes. A coefficient profile plot was produced against the log $(L)$ sequence. Gene set enrichment analysis for NPTX1 (C), PCSK1 (D), ASXL3 (E), and TRIM9 (F). The top four pathways enriched in the high expression group are shown. 

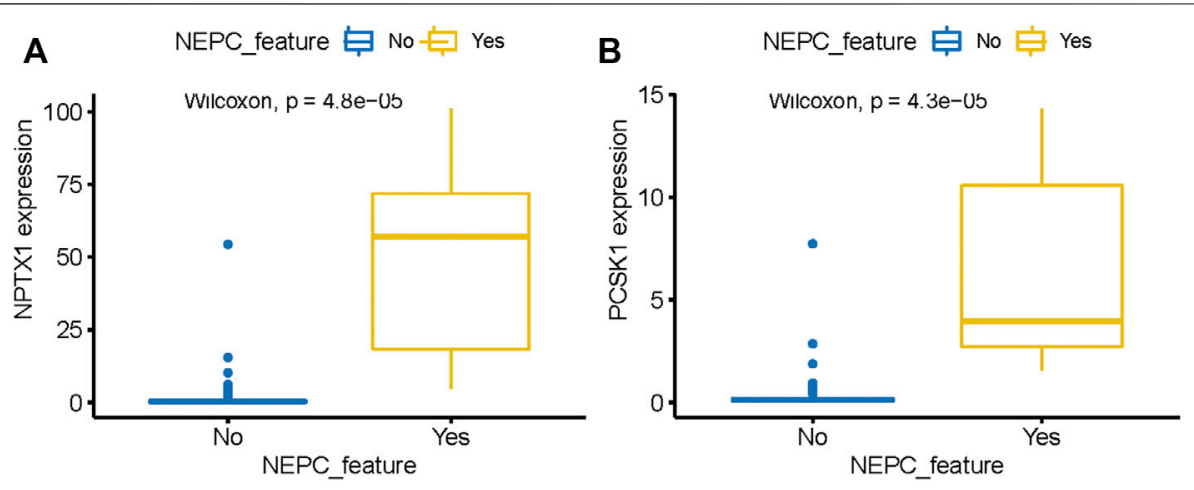

C NEPC_feature 官 No ウ Yes D

D NEPC_feature 官 No 官 Yes
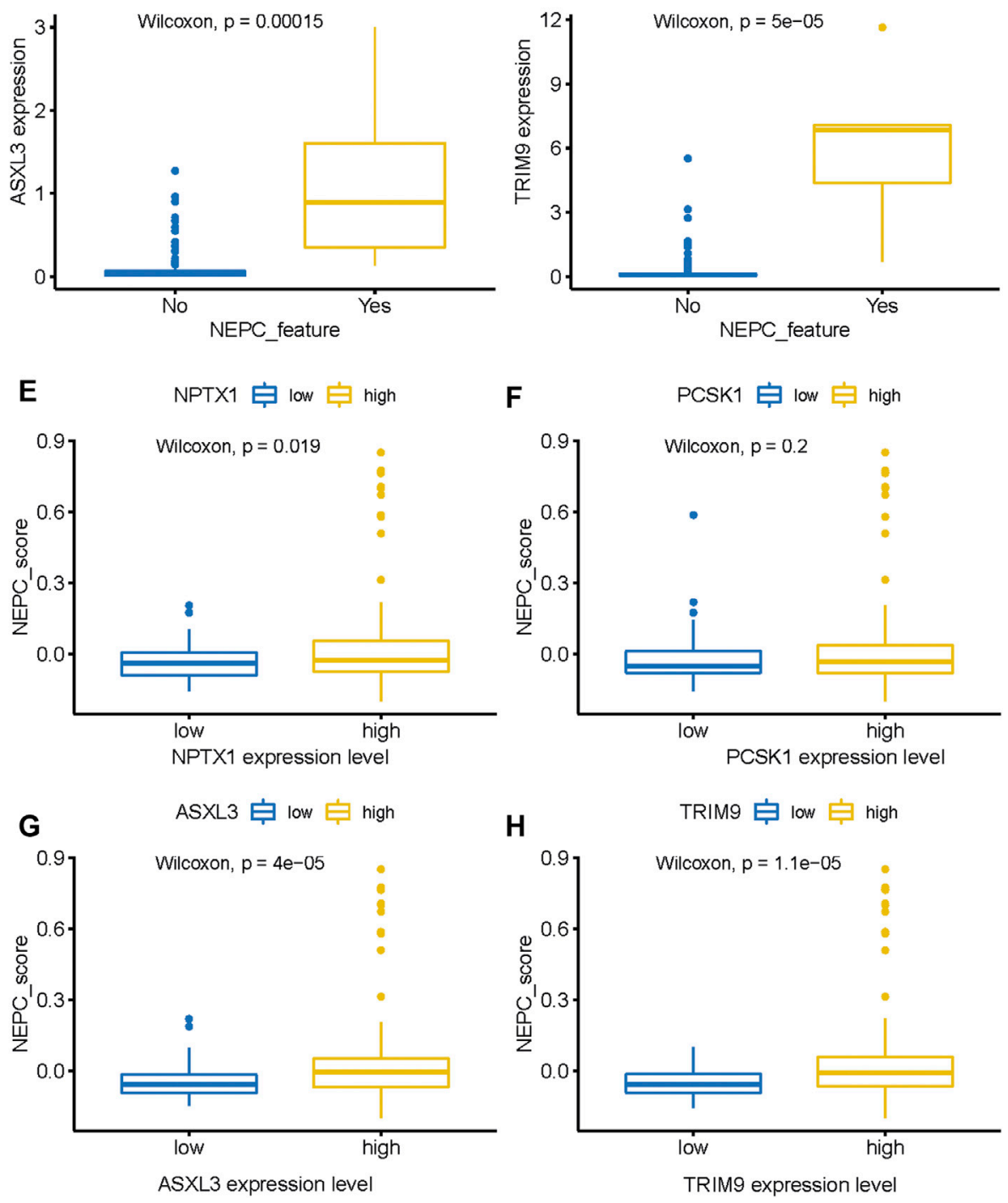

FIGURE 6 | The expression level of four genes between samples with NEPC feature or not from the SU2C dataset. NPTX1 (A), PCSK1 (B), ASXL3 (C), and TRIM9 (D) expression level in samples with $(\mathrm{N}=6)$ or without $(\mathrm{N}=161)$ NEPC feature. The relationship between the expression level of identified biomarkers [NPTX1 (E), PCSK1 (F), ASXL3 (G), and TRIM9 (H)] and the NEPC score. The median value of gene expression level was used as a cutoff to classify samples into high- (N = 104) and low- $(\mathrm{N}=104)$ expression level groups. 
TABLE 2 | The coefficients of identified genes in the NEPC signature.

\begin{tabular}{lcc}
\hline Gene & Coefficient & $\boldsymbol{p}$-value \\
\hline NPTX1 & 0.003247 & 0.028 \\
PCSK1 & 0.003142 & 0.011 \\
ASXL3 & 0.024173 & 0.028 \\
TRIM9 & 0.020398 & 0.011
\end{tabular}

of the NEPC prediction was 0.995 , showing its strong plausibility as the NEPC diagnosis signature (Figure 7B).

\section{Validation of the Established NEPC Signature}

Next, in the validation dataset of an independent NEPC program (nepc_wcm_2016), it was noted that patient samples with NEPC features (i.e., the CRPC-NE samples) had significantly higher expression levels of the four genes compared with the CRPC-adeno patient samples $(p<0.001$, Figures 8A-D). Furthermore, the NEPC samples in the validation cohort (nepc_wcm 2016) also had significantly higher predicted signature scores compare with those in the CRPCadeno samples (Figure 8E). Meanwhile, the AUC of the NEPC prediction was 0.833 , which confirmed the validity of this model in terms of its accuracy for the diagnosis of NEPC (Figure 8F).

\section{GENOMIC DIFFERENCES BETWEEN SAMPLES WITH HIGH AND LOW NEPC SIGNATURE SCORES}

The top 20 mutated genes in samples with high and low NEPC signature scores from the SU2C-PCF dataset were shown in Figures
9A, B. No significant differences were identified in the prevalence of altered genes comparing between these two groups, including genes associated with the AR signaling pathway ( $A R$ and FOXA1). However, in the validation dataset, a significantly higher prevalence of certain genes, including RB1, METTL24, and ADRM1, were identified in the samples with a high NEPC signature score (Figure 9C). On the other hand, KPRP and SPOP were identified as more prevalent genes in the CRPC-adeno samples (Figure 9D).

\section{DISCUSSION}

NEPC is a highly aggressive subtype of prostate cancer that is associated with poor survival and developing resistance to novel hormone treatments, including the administration of abiraterone and enzalutamide. Early diagnosis of NEPC and the timely application of treatment for advanced NEPC are difficult to accomplish, however, due to a deficiency of robust molecular biomarkers [20]. The previously established NEPC score, which was comprehensively analyzed on the basis of DNA alteration, methylation, and RNA expression parameters, has been shown to enable the precise identification of CRPC-NE $[8,9]$. Although this model was effective, however, it was not appropriate for clinical popularization or application due to its costliness and its complex algorithm. In order to find a concise but effective diagnosis model for NEPC, the present study aimed to analyze the RNA-seq data of therapy-related NEPC compared with CRPC.

Biological analysis of our identified gene modules has suggested that neurite extension, as the neuroendocrine marker, is a highly correlated pathway in the modules. Changes in membrane potential and ion channel activity may occur during the process of NE differentiation in which the overexpression of alpha $(1 \mathrm{H})$ mRNA (i.e., a single type of LVA calcium channel mRNA) was reported during neuroendocrine
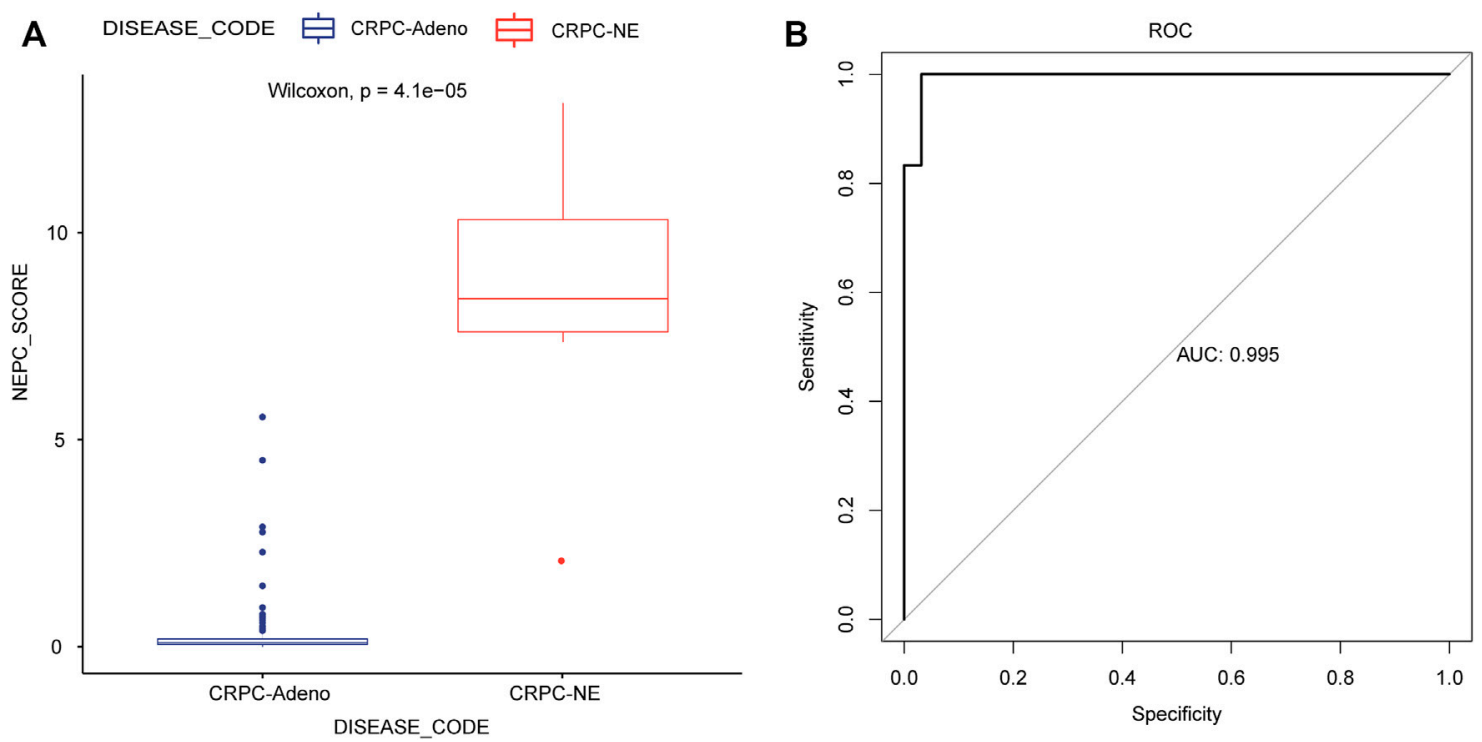

FIGURE 7 | Application and evaluation of the NEPC predicted signature model. (A) The differences in predicted signature score between CRPC-NE and CRPCadeno samples. (B) The ROC curves for NEPC predicted signature model in the training cohort. 

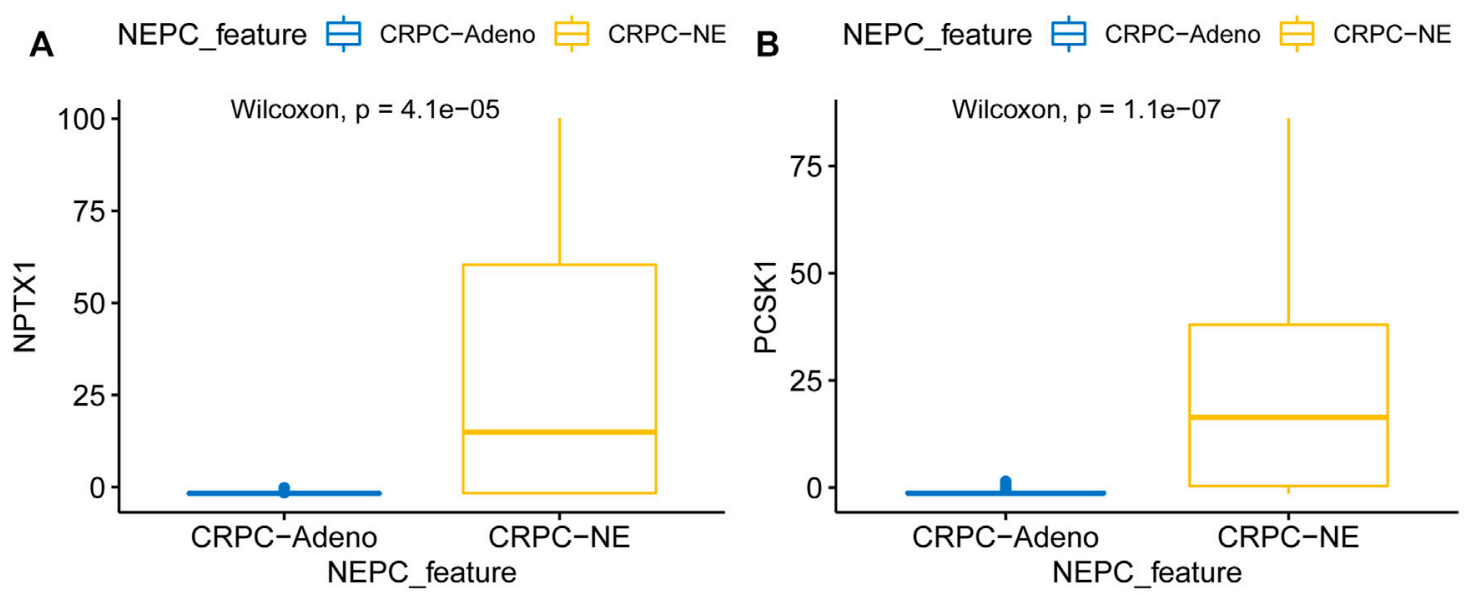

C NEPC_feature 追 CRPC-Adeno

D NEPC_feature 官 CRPC-Adeno CRPC-NE
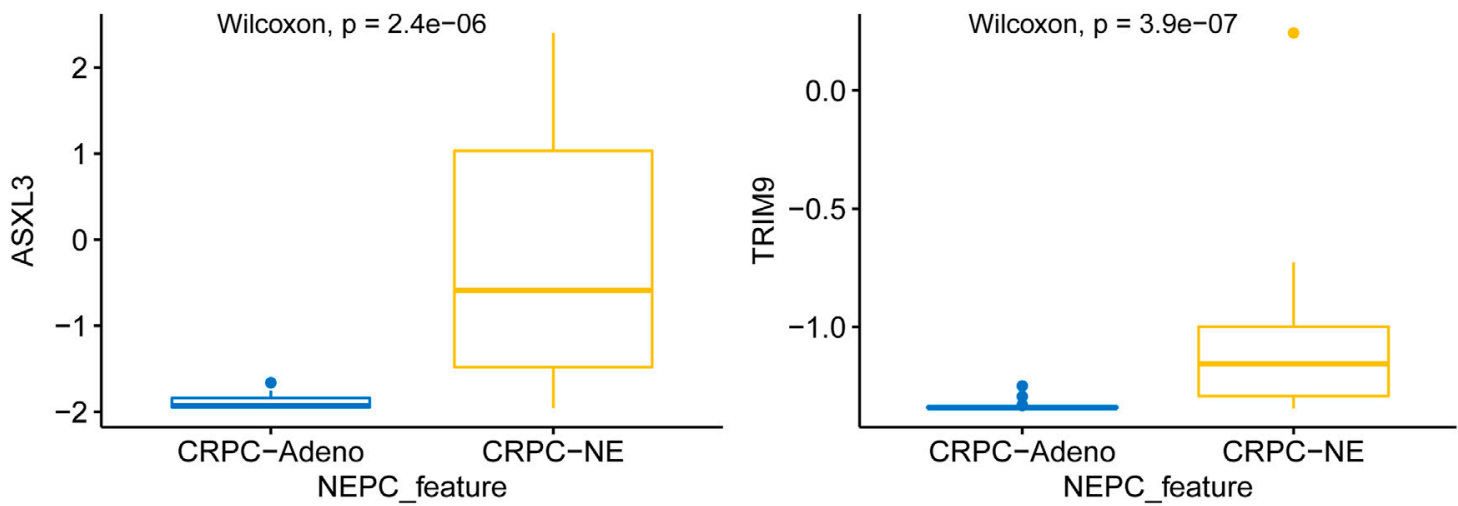

E DISEASE_CODE 官 CRPC-Adeno 追 CRPC-NE

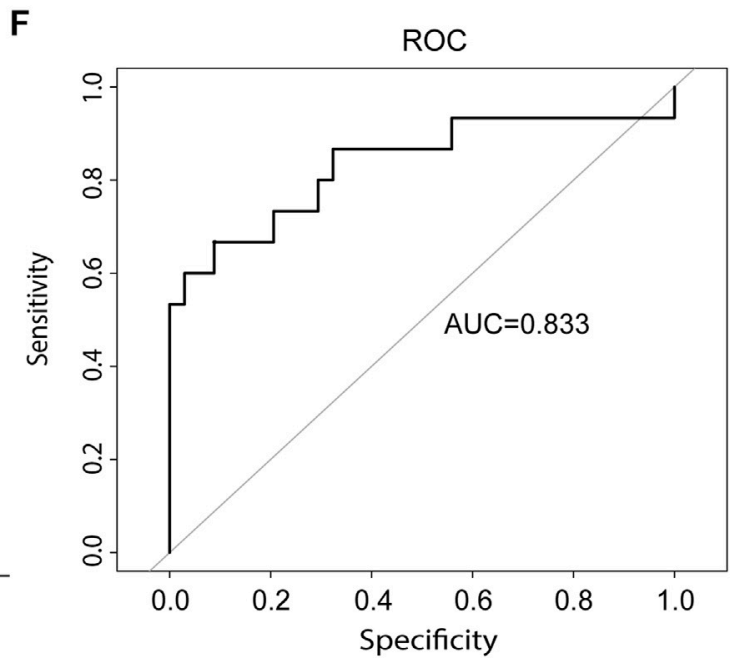

FIGURE 8 | Model validation using the lasso method in an independent dataset. The mRNA differential expression analysis of four potential predicting genes between the samples with $(\mathrm{N}=15)$ or without $(\mathrm{N}=34) \mathrm{NE}$ feature in the nepc_wcm_2016 dataset. (A) NPTX1. (B) PCSK1. (C) ASXL3. (D) TRIM9. (E) The differences in predicted signature score between NEPC and Adenocarcinoma samples. (F) NEPC feature-dependent ROC curves were performed in the validation cohort.

differentiation [21]. Furthermore, calcium channels have been shown to facilitate neurite lengthening via promoting basal calcium entry at the resting membrane potential [22-24].
Ultimately, four novel biomarkers for NEPC were identified, including NPTX1, PCSK1, ASXL3, and TRIM9, by LASSO regression analysis, and it was noteworthy that 

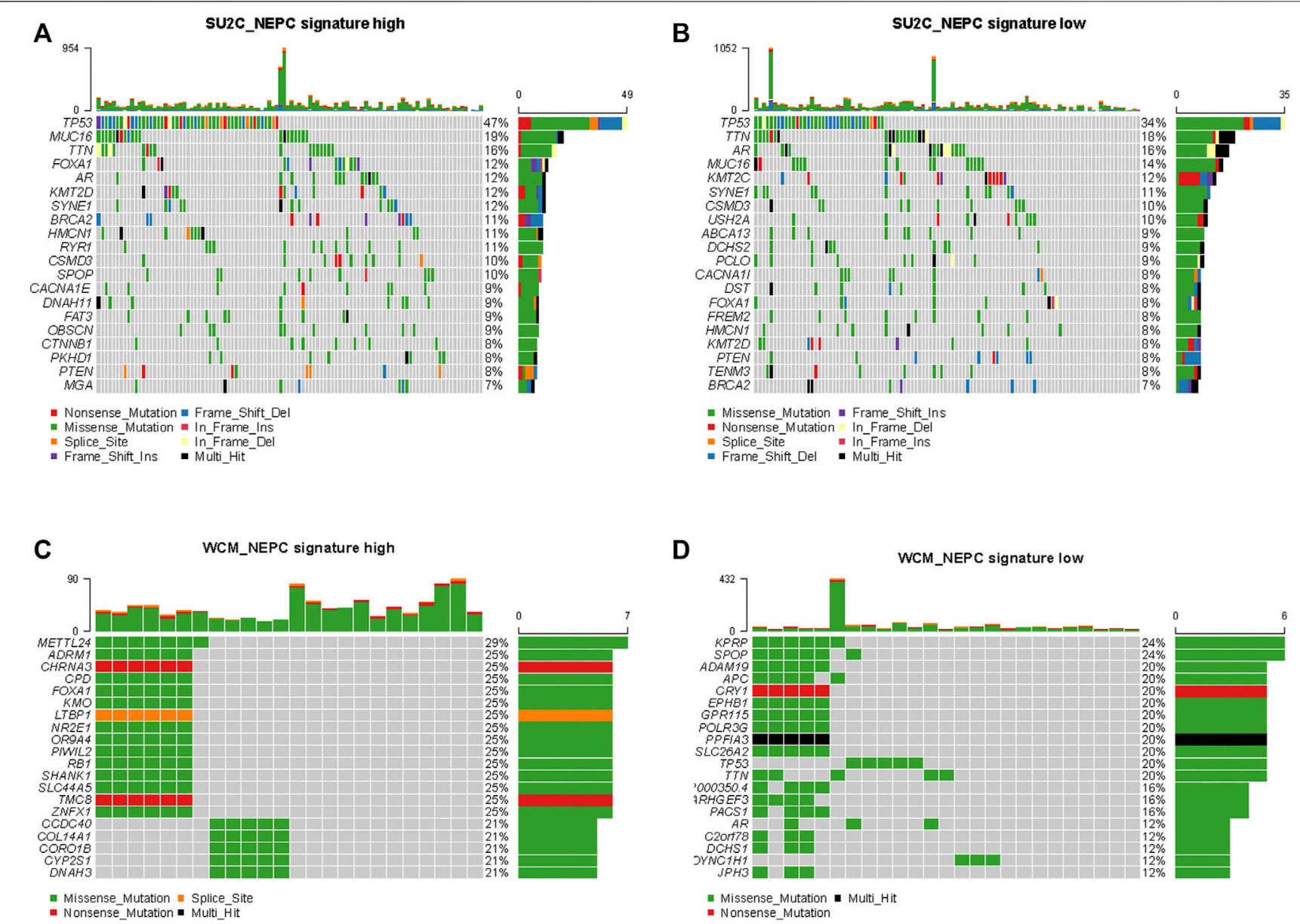

FIGURE 9 | Genomic alterations in samples with high and low NEPC signature. The oncoprint results of samples with high (A) and low (B) NEPC signature of SU2C dataset. The oncoprint results of samples with high (C) and low (D) NEPC signature of WCM dataset. The top 20 most prevalent genes were presented.

some of these genes have already been previously identified in the NEPC. Through exon array profiling, Tsai and others identified that $87 \%(13 / 16)$ of NEPC patients had outlier expression of NPTX1 and PCSK1, findings that were also in support of the strong plausibility of these two candidates as markers for the diagnosis of NEPC [25]. NPTX1 encoded a secreted glycoprotein of size $47-50 \mathrm{kDa}$ and was first identified in the central nervous system as a member of the pentraxin family [26]. Based on mass-spectrometry-based proteomics, it was identified that the level of NPTX1, which is involved in neurogenesis, was elevated in NEPC samples [27]. Recently, an increasing number of studies have shown that NPTX1 is involved in the progression of various cancers, including lung cancer [28], colon cancer [29], and gastric cancer [30]. PCSK1, encoding prohormone convertase 1 , belongs to the proprotein convertase family, and its overexpression has been revealed in various subtypes of neuroendocrine tumors [31-33]. Previous studies have shown that treatment-related NEPC also exhibits a high expression of PCSK1, and the pattern of promoter methylation was observed to be different among distinct phenotypes of PCa [34, 35]. On the other hand, the role of ASXL3 and TRIM9 in the diagnosis of NEPC has not been previously shown. ASXL3, the polycomb group (PcG) protein, is essential for neuroendocrine (NE) lung cancer development and was shown via KEGG analysis to be associated with the multiple neuron differentiation signaling pathways [36, 37]. Furthermore, ASXL3 was found to serve as an effective biomarker for predicting the sensitivity towards BET protein inhibitors in small cell lung cancer (SCLC), highlighting its potential as an actionable biomarker [38, 39]. TRIM9, encoding a brain-specific E3 ubiquitin ligase, has been shown by GO analysis to be involved in the neurological disease and inflammation pathways [40]. Its overexpression promotes cell proliferation, and inhibits cell apoptosis via the NF- $\kappa$ B signaling pathway in uterine leiomyoma [41]. As an important transcription factor, $N F-\kappa B$ has also been implicated in the acquisition of neuroendocrine characteristics in prostate cancer cells [42].

Although several diagnosis markers have now been identified, histological assessment with neuroendocrine differentiation remains "the gold standard" for clinical diagnosis of NEPC. However, the clinical and cellular heterogeneity of NEPC patients causes many difficulties for the clinical management of NEPC, and the sampling process of 
histological assessment is both invasive and painful to patients [43]. Recently, comprehensive genomic profiling revealed molecular heterogeneity in the genomic landscape of metastatic CRPC [8,9]. The results of the present study should prove to be of great benefit in terms of improving clinical applicability. Furthermore, the novel NEPC signature that has been identified will have the advantage of overcoming the heterogeneity that leads to inaccuracies in the diagnosis of patients with NEPC. Finally, the high AUC value that was determined provides confirmatory evidence that the NEPC signature is trustworthy in terms of its putative role as a novel diagnostic tool to distinguish samples of patients with CRPCNE from those with CRPC-adeno. However, it should be acknowledged that the present study has some limits because of the sample sizes. Moreover, this study was conducted based on public databases. Therefore, we having been collecting the NEPC patient samples in our hospital and would validate the NEPC signature scoring system as a diagnostic tool in an independent cohort. At the same time, the merits of this NEPC signature scoring system would be assessed with regard to its application in the clinic. To corroborate these findings, the functional roles of the identified four hub genes should be explored in relevant cell lines or in a mouse model. Furthermore, other biomarkers, such as low expression of androgen-regulated genes [e.g., KLK3 (PSA), TMPRSS2, and NXK3.1] and high expression of neuroendocrine-associated genes (e.g., CGA and $S Y P$ ), may be investigated subsequently to provide further confirmatory evidence for the accuracy of the NEPC signature in the diagnosis of NEPC.

In conclusion, the present study has investigated co-expressed gene modules that were highly correlated with the NEPC score, and four hub genes were screened. The findings obtained have improved our understanding of the underlying molecular mechanism of NEPC. As a model, these hub genes could represent a novel diagnostic marker and therapeutic target for NEPC.

\section{DATA AVAILABILITY STATEMENT}

The datasets presented in this study can be found in online repositories. The names of the repository/repositories and accession number(s) can be found in the article/ Supplementary Material.

\section{REFERENCES}

1. MaryBeth BC, Isabelle S, Jason AE, Freddie B, and Ahmedin J. Recent Global Patterns in Prostate Cancer Incidence and Mortality Rates. Amsterdam, Netharlands: European Urology (2019).

2. Bray F, Ferlay J, Soerjomataram I, Siegel RL, Torre LA, and Jemal A. Global Cancer Statistics 2018: GLOBOCAN Estimates of Incidence and Mortality Worldwide for 36 Cancers in 185 Countries. CA: A Cancer J Clinicians (2018) 68(6):394-424. doi:10.3322/caac.21492

3. Rebello RJ, Oing C, Knudsen KE, Loeb S, Johnson DC, Reiter RE, et al. Prostate Cancer. Nat Rev Dis Primers (2021) 7(1):9. doi:10.1038/s41572-020-00243-0

\section{ETHICS STATEMENT}

The studies involving human participants were reviewed and approved by the Biomedical Research Ethics Committee of Peking University First Hospital. The patients/participants provided their written informed consent to participate in this study.

\section{AUTHOR CONTRIBUTIONS}

CZ: data collection, drafting the article, design of this work, and data analysis; JQ: data analysis and manuscript revision; YW: data collection and analysis; ZZ: data collection; WY: data collection; YG: data collection; XL: data collection; $\mathrm{ZH}$ : data collection; LZ: data collection.

\section{FUNDING}

The present study was funded by The National Key R and D Program of China (grant no. 2019YFA0906000) and The National Natural Science Foundation of China (grant nos. 81772703 and 81972380).

\section{CONFLICT OF INTEREST}

The authors declare that the research was conducted in the absence of any commercial or financial relationships that could be construed as a potential conflict of interest.

\section{ACKNOWLEDGMENTS}

We are grateful of all participants in Peking University First Hospital.

\section{SUPPLEMENTARY MATERIAL}

The Supplementary Material for this article can be found online at: https://www.por-journal.com/articles/10.3389/pore.2021.1609968/ full\#supplementary-material

4. Yamada Y, and Beltran H. Clinical and Biological Features of Neuroendocrine Prostate Cancer. Curr Oncol Rep (2021) 23(2):15. doi:10.1007/s11912-02001003-9

5. Vincenza C, Clara O, Kenneth WE, Rohan B, Michael S, Ana M, et al. Clinical Features of Neuroendocrine Prostate Cancer. Eur J Cancer (2019).

6. Aparicio AM, Harzstark AL, Corn PG, Wen S, Araujo JC, Tu S-M, et al.PlatinumBased Chemotherapy for Variant Castrate-Resistant Prostate Cancer. Clin Cancer Res (2013) 19(13):3621-30. doi:10.1158/1078-0432.ccr-12-3791

7. Bhagirath D, Liston M, Akoto T, Lui B, Bensing BA, Sharma A, et al. Novel, Non-invasive Markers for Detecting Therapy Induced Neuroendocrine Differentiation in Castration-Resistant Prostate Cancer Patients. Sci Rep (2021) 11(1):8279. doi:10.1038/s41598-021-87441-2 
8. Beltran H, Prandi D, Mosquera JM, Benelli M, Puca L, Cyrta J, et al. Divergent Clonal Evolution of Castration-Resistant Neuroendocrine Prostate Cancer. Nat Med (2016) 22(3):298-305. doi:10.1038/nm.4045

9. Abida W, Cyrta J, Heller G, Prandi D, Armenia J, Coleman I, et al. Genomic Correlates of Clinical Outcome in Advanced Prostate Cancer. Proc Natl Acad Sci USA (2019) 116(23):11428-36. doi:10.1073/pnas.1902651116

10. Bakht MK, Derecichei I, Li Y, Ferraiuolo RM, Dunning M, Oh SW, et al. Neuroendocrine Differentiation of Prostate Cancer Leads to PSMA Suppression. Endocr Relat Cancer (2018) 26(2):131-46. doi:10.1530/ERC18-0226

11. Epstein JI, Amin MB, Beltran H, Lotan TL, Mosquera J-M, Reuter VE, et al. Proposed Morphologic Classification of Prostate Cancer with Neuroendocrine Differentiation. Am J Surg Pathol (2014) 38(6):756-67. doi:10.1097/ pas. 0000000000000208

12. Lovnicki J, Gan Y, Feng T, Li Y, Xie N, Ho C-H, et al. LIN28B Promotes the Development of Neuroendocrine Prostate Cancer. J Clin Invest (2020) 130(10): 5338-48. doi:10.1172/jci135373

13. Tiwari R, Manzar N, Bhatia V, Yadav A, Nengroo MA, Datta D, et al. Androgen Deprivation Upregulates SPINK1 Expression and Potentiates Cellular Plasticity in Prostate Cancer. Nat Commun (2020) 11(1):384. doi:10.1038/s41467-019-14184-0

14. Yamada Y, and Beltran H. Clinical and Biological Features of Neuroendocrine Prostate Cancer. Curr Oncol Rep (2021) 23(2):15. doi:10.1007/s11912-02001003-9

15. Aggarwal RR, and Small EJ. Small-cell/neuroendocrine Prostate Cancer: a Growing Threat? Oncology (Williston Park) (2014) 28(10):838-40.

16. Beltran H, Prandi D, Mosquera JM, Benelli M, Puca L, Cyrta J, et al. Divergent Clonal Evolution of Castration-Resistant Neuroendocrine Prostate Cancer. Nat Med (2016) 22(3):298-305. doi:10.1038/nm.4045

17. Qiuli L, Jian P, Lin-ang W, Zhuowei H, Jing X, Xingxia Y, et al. Histone Demethylase PHF8 Drives Neuroendocrine Prostate Cancer Progression by Epigenetically Upregulating FOXA2. J Pathol (2020)(1) 253106-118. doi:10.1002/path.5557

18. Sauerbrei W, Royston P, and Binder H. Selection of Important Variables and Determination of Functional Form for Continuous Predictors in Multivariable Model Building. Statist Med (2007) 26(30):5512-28. doi:10.1002/sim.3148

19. Subramanian A, Tamayo P, Mootha VK, Mukherjee S, Ebert BL, Gillette MA, et al. Gene Set Enrichment Analysis: a Knowledge-Based Approach for Interpreting Genome-wide Expression Profiles. Proc Natl Acad Sci (2005) 102(43):15545-50. doi:10.1073/pnas.0506580102

20. Dong B, Miao J, Wang Y, Luo W, Ji Z, Lai H, et al. Single-cell Analysis Supports a Luminal-Neuroendocrine Transdifferentiation in Human Prostate Cancer. Commun Biol (2020) 3(1):778. doi:10.1038/s42003020-01476-1

21. Mariot P, Vanoverberghe K, Lalevée N, Rossier MF, and Prevarskaya N. Overexpression of an a1H (Cav3.2) T-type Calcium Channel during Neuroendocrine Differentiation of Human Prostate Cancer Cells. J Biol Chem (2002) 277(13):10824-33. doi:10.1074/jbc.m108754200

22. Fukami K, Sekiguchi F, Yasukawa M, Asano E, Kasamatsu R, Ueda M, et al. Functional Upregulation of the H2S/Cav3.2 Channel Pathway Accelerates Secretory Function in Neuroendocrine-Differentiated Human Prostate Cancer Cells. Biochem Pharmacol (2015) 97(3):300-9. doi:10.1016/ j.bcp.2015.08.005

23. Lemonnier L, Lazarenko R, Shuba Y, Thebault S, Roudbaraki M, Lepage G, et al. Alterations in the Regulatory Volume Decrease (RVD) and SwellingActivated Cl-Current Associated with Neuroendocrine Differentiation of Prostate Cancer Epithelial Cells. Endocr Relat Cancer (2005) 12(2):335-49. doi:10.1677/erc. 1.00898

24. Fletcher PA, Sherman A, and Stojilkovic SS. Common and Diverse Elements of Ion Channels and Receptors Underlying Electrical Activity in Endocrine Pituitary Cells. Mol Cell Endocrinol (2018) 463:23-36. doi:10.1016/ j.mce.2017.06.022

25. Tsai HK, Lehrer J, Alshalalfa M, Erho N, Davicioni E, and Lotan TL. Gene Expression Signatures of Neuroendocrine Prostate Cancer and Primary Small Cell Prostatic Carcinoma. BMC cancer (2017) 17(1):759. doi:10.1186/s12885017-3729-z
26. Boles NC, Hirsch SE, Le S, Corneo B, Najm F, Minotti AP, et al. NPTX1 Regulates Neural Lineage Specification from Human Pluripotent Stem Cells. Cel Rep (2014) 6(4):724-36. doi:10.1016/j.celrep.2014.01.026

27. Amilcar F-M, Tobias BB, Charlotte L, Tanveer SB, Dong L, Mads L, et al. Proteogenomic Characterization of Patient-Derived Xenografts Highlights the Role of REST in Neuroendocrine Differentiation of Castration-Resistant Prostate Cancer. Clin Cancer Res (2018) 25(2):595-608. doi:10.1158/10780432.CCR-18-0729

28. Zhou C, Qin Y, Xie Z, Zhang J, Yang M, Li S, et al. NPTX1 Is a Novel Epigenetic Regulation Gene and Associated with Prognosis in Lung Cancer. Biochem Biophysical Res Commun (2015) 458(2):381-6. doi:10.1016/j.bbrc.2015.01.124

29. Peng X, Pan K, Zhao W, Zhang J, Yuan S, Wen X, et al.NPTX1 Inhibits colon Cancer Cell Proliferation through Down-Regulating Cyclin A2 and CDK2 Expression. Cell Biol Int (2018) 42(5):589-97. doi:10.1002/cbin.10935

30. Yan H, Zheng C, Li Z, Bao B, Yang B, Hou K, et al.NPTX1 Promotes Metastasis via Integrin/FAK Signaling in Gastric Cancer. Cmar (2019) Vol. 11:3237-51. doi:10.2147/cmar.s196509

31. Yue W, Wang T, Zachariah E, Lin Y, Yang CS, Xu Q, et al. Transcriptomic Analysis of Pancreatic Cancer Cells in Response to Metformin and Aspirin: an Implication of Synergy. Sci Rep (2015) 5(1):13390. doi:10.1038/srep13390

32. Kidd M, Modlin IM, and Drozdov I. Gene Network-Based Analysis Identifies Two Potential Subtypes of Small Intestinal Neuroendocrine Tumors. Bmc Genomics (2014) 15:595. doi:10.1186/1471-2164-15-595

33. Jansen E, Ayoubi TAY, Meulemans SMP, and Van de Ven WJM. Neuroendocrine-specific Expression of the Human Prohormone Convertase 1 Gene. J Biol Chem (1995) 270(25):15391-7. doi:10.1074/jbc.270.25.15391

34. Ramalingam S, Eisenberg A, Foo WC, Freedman J, Armstrong AJ, Moss LG, et al.Treatment-related Neuroendocrine Prostate Cancer Resulting in Cushing's Syndrome. Int J Urol (2016) 23(12):1038-41. doi:10.1111/iju.13225

35. Brennen WN, Yezi Z, Ilsa MC, Susan LD, Lizamma A, Radhika AP, et al.Resistance to Androgen Receptor Signaling Inhibition Does Not Necessitate Development of Neuroendocrine Prostate Cancer. JCI Insight 6(8):e146827. doi:10.1172/jci.insight.146827

36. Katoh M. Functional and Cancer Genomics of ASXL Family Members. $\mathrm{Br}$ J Cancer (2013) 109(2):299-306. doi:10.1038/bjc.2013.281

37. Shukla V, Rao M, Zhang H, Beers J, Wangsa D, Wangsa D, et al. ASXL3 Is a Novel Pluripotency Factor in Human Respiratory Epithelial Cells and a Potential Therapeutic Target in Small Cell Lung Cancer. Cancer Res (2017) 77(22):6267-81. doi:10.1158/0008-5472.can-17-0570

38. Rossi G, Bertero L, Marchiò C, and Papotti M. Molecular Alterations of Neuroendocrine Tumours of the Lung. Histopathology (2018) 72(1):142-52. doi:10.1111/his.13394

39. Szczepanski AP, Zhao Z, Sosnowski T, Goo YA, Bartom ET, and Wang L. ASXL3 Bridges BRD4 to BAP1 Complex and Governs Enhancer Activity in Small Cell Lung Cancer. Genome Med (2020) 12(1):63. doi:10.1186/s13073020-00760-3

40. Zeng J, Wang Y, Luo Z, Chang L-C, Yoo JS, Yan H, et al. TRIM9-Mediated Resolution of Neuroinflammation Confers Neuroprotection upon Ischemic Stroke in Mice. Cel Rep (2019) 27(2):549-60.546. doi:10.1016/ j.celrep.2018.12.055

41. Yang F, Liu H, Yu Y, and Xu L. TRIM9 Overexpression Promotes Uterine Leiomyoma Cell Proliferation and Inhibits Cell Apoptosis via NF-Kb Signaling Pathway. Life Sci (2020) 257:118101. doi:10.1016/j.lfs.2020.118101

42. Amorino GP, and Parsons SJ. Neuroendocrine Cells in Prostate Cancer. Crit Rev Eukaryot Gene Expr (2004) 14(4):287-300. doi:10.1615/ critreveukaryotgeneexpr.v14.i4.40

43. McNally CJ, Ruddock MW, Moore T, and McKenna DJ. Biomarkers that Differentiate Benign Prostatic Hyperplasia from Prostate Cancer: A Literature Review. Cmar (2020) Vol. 12:5225-41. doi:10.2147/cmar.s250829

Copyright $\odot 2021$ Zhang, Qian, Wu, Zhu, Yu, Gong, Li, He and Zhou. This is an open-access article distributed under the terms of the Creative Commons Attribution License (CC BY). The use, distribution or reproduction in other forums is permitted, provided the original author(s) and the copyright owner(s) are credited and that the original publication in this journal is cited, in accordance with accepted academic practice. No use, distribution or reproduction is permitted which does not comply with these terms. 